\title{
Optimized response to electricity time-of-use tariff of a compressed natural gas fuelling station ${ }^{1}$
}

\author{
Charles Kagiri*, Evan M. Wanjiru, Lijun Zhang, Xiaohua Xia \\ Centre of New Energy Systems, Department of Electrical, Electronic and Computer Engineering, University of \\ Pretoria, Pretoria 0002, South Africa
}

\begin{abstract}
Compressed natural gas propulsion of vehicles has been shown to have advantages over petrol and diesel propulsion due to lower carbon dioxide emissions as well as the increased durability of vehicle engines. The growth of compressed natural gas as an alternative fuel to petrol and diesel can be accelerated by implementing strategies that result in the economical operation of the distribution infrastructure. Economic scheduling of power consumption is a useful strategy for reducing the cost of energy for both industrial and domestic consumers who operate in time-of-use based electricity pricing environments. In this paper, an optimal energy management strategy is proposed for the operation of a compressed natural gas fuelling station. The compressor energy consumption, being the main component of the total operating cost of the fuelling station, presents a cost saving opportunity through which optimal scheduling of operation can be used to lower cost of operation of the station. The developed model shows potential average savings of 59.3\% in daily electricity costs while maximizing compressor life through minimization of compressor cycling.
\end{abstract}

Keywords: Compressed Natural Gas, Fast-fill, Demand response, Optimization

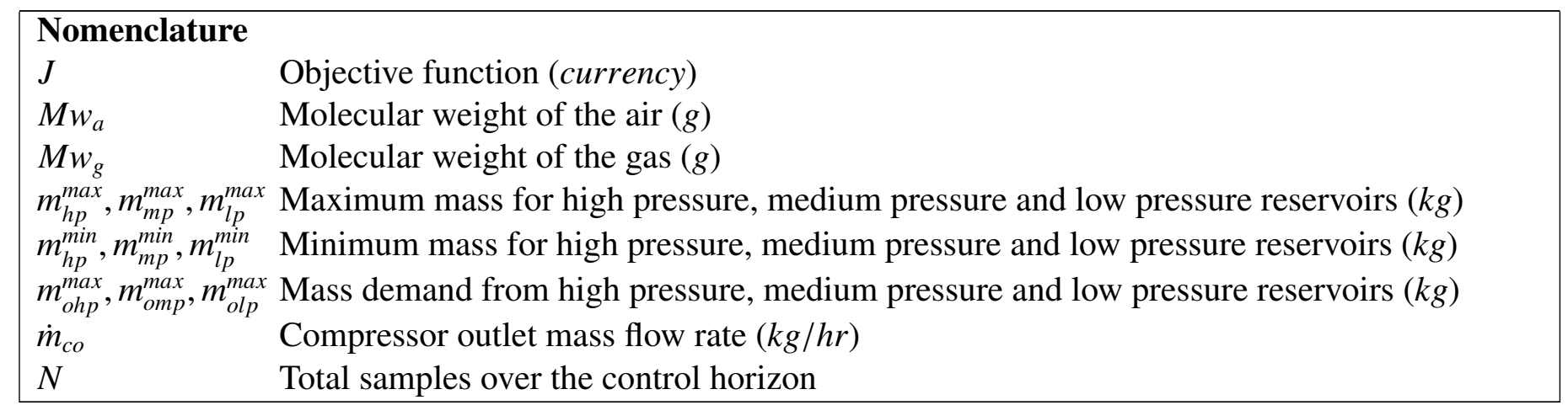

\footnotetext{
${ }^{1}$ The short version of the paper was presented at ICAE2017, Aug 21-24, Cardiff, UK. This paper is a substantial extension of the short version of the conference paper.

${ }^{*}$ Corresponding author. Tel. +27 81547 6363; Fax +27 123625000.

Email addresses: kagiricharles@gmail.com(Charles Kagiri ), lijun.zhang@up.ac.za (Lijun Zhang)
} 


\begin{tabular}{|ll|}
\hline$n$ & Gas quantity (moles) \\
$p$ & Pressure (bars) \\
$p_{c o}$ & Compressor motor power rating $(k W)$ \\
$p_{e}$ & Price of electricity under TOU tarrif $($ currency $/ \mathrm{kWh})$ \\
$p_{h p}^{\max }, p_{m p}^{\max }, p_{l p}^{\max }$ & Maximum pressure for high pressure, medium pressure and low pressure reservoirs $($ bars $)$ \\
$p_{h p}^{\min }, p_{m p}^{\min }, p_{l p}^{\min }$ & Minimum pressure for high pressure, medium pressure and low pressure reservoirs $($ bars $)$ \\
$Q_{s t d}$ & Capacity of the compressor under standard conditions $\left(\mathrm{Nm}^{3} / \mathrm{hr}\right)$ \\
$R$ & Universal gas constant $($ Lbar $/ \mathrm{Kmol})$ \\
$T$ & Absolute temperature $(\mathrm{K})$ \\
$u$ & State of compressor switch \\
$u_{h p}, u_{m p}, u_{l p}$ & State of reservoir valves for high pressure, medium pressure and low pressure reservoirs \\
$V$ & Volume of cascade reservoir tanks $($ Litres $)$ \\
$z$ & Compressibility factor of CNG \\
$\rho_{a, s t d}$ & Density of air under standard conditions $\left(\mathrm{kg} / \mathrm{m}^{3}\right)$ \\
\hline
\end{tabular}

\section{Introduction}

Compressed Natural Gas (CNG) is one of the alternatives to liquid hydrocarbon fuels that have been promoted to address the challenges of air pollution, energy dependence and climate change $[1,2,3,4]$. CNG, which is largely made up of methane and small quantities of other hydrocarbons such as propane butane and ethane could be considered a clean fuel in comparison with gasoline and diesel because it has the lowest emissions among hydrocarbon fuels [5, 6]. Furthermore, CNG vehicles have been shown to have lower total cost of ownership (TCO) than gasoline or diesel fuelled cars [7, 8, 9]. In recent years, the use of CNG for vehicle propulsion has been increasing worldwide in both developed and developing countries, especially in countries that have suffered severe air pollution from rapid industrialization in the past three decades such as India and China [10, 11]. The expanding adoption of CNG has corresponded to a simultaneous growth of CNG distribution infrastructure for vehicular end users [12]. In South Africa for example, the Department of Energy recognizes compressed natural gas as one of the possible energy options for transportation that will contribute to the reduction of the country's carbon footprint [13]. In view of the long way to large-scale adoption of electric vehicles, CNG is viewed as an appropriate transition fuel towards a greener transportation sector [14]. Introduction of public service vehicles powered by CNG as well as growth in the number of CNG fleet customers has resulted in the growth in number of vehicle fuelling station in the city of Johannesburg and Pretoria. Being consumers of electric power, CNG fuelling stations are subject to the availability and pricing conditions of the electricity environment in which they operate [15]. While the expansion of CNG distribution infrastructure is a sign of investor confidence in the future of the industry, the distribution infrastructure is subject to challenges arising from the supply of electricity and must implement adaptive strategies to remain energy efficient and economically attractive.

CNG is stored under high pressure in on-board vehicle tanks, from where it flows to the combustion engine under regulation [16]. CNG powered vehicles receive their fuel from high pressure reservoirs at $\mathrm{CNG}$ fuelling stations. Although refuelling of vehicles with natural gas can take a 
long time, the fast-fill process which is used at most $\mathrm{CNG}$ fuelling stations has been developed to achieve fuelling times of less than five minutes, which is comparable to the fuelling time of diesel or gasoline powered automobiles [17]. Fast-fill fuelling stations use reservoir tanks in a cascaded storage system divided into low pressure, medium pressure and high pressure levels [18]. The dispenser at the fast-fill station has electronic sequencing valves that are controlled by a microprocessor algorithm as well as sensors for measuring mass flow from each of the three reservoirs [19]. A vehicle typically arrives with low pressure in its tank and the dispenser starts the filling of the tank by connecting it to the low pressure reservoir. The differential pressure causes gas to flow into the vehicle tank and as the vehicle tank fills up, the mass flow rate between the reservoir and the vehicle tank falls to a limit after which the dispenser switches the filling to the medium pressure reservoir for a higher mass flow rate [20]. The vehicle tank continues to fill up from the medium pressure reservoir which results in the mass flow rate falling until a limit is reached and the dispenser switches the filling from the medium pressure reservoir to the high pressure reservoir. The high pressure reservoir completes the filling of the vehicle tank [21]. It is possible in some scenarios for the vehicle to arrive with a high tank pressure that can only be filled from the high pressure reservoir since it is almost full or from the medium pressure reservoir followed by the high pressure reservoir. The dispenser algorithm determines from initial vehicle tank pressure which reservoir to start with [22]. It is also possible in some scenarios for the customer to request a quantity of gas that does not result in filling of the vehicle tank and therefore receive gas from the low pressure reservoir and medium pressure reservoir only, or even the low pressure reservoir alone. This means that the demand of gas from one reservoir is not always synchronized with demand from the other two reservoirs. The CNG station dispenser runs a vehicle filling algorithm that is compensated for temperature and pressure to ensure that correct quantities of gas demanded by consumer are dispensed to the vehicle tank. This isolates the consumer vehicle tank from the fluctuations in the pressure and temperature that may occur in the cascade storage as a result of vehicle gas demand itself or as a result of operation control [23, 24, 25, 26, 27].

A priority panel controls the filling of the three reservoirs of the cascade storage, by switching the gas flowing from the outlet of the compressor between the reservoir valves [28]. The priority panel is operated through a PLC, which runs an algorithm that controls the sequence of opening the three reservoir valves during charging of the cascade storage by the reciprocating compressor [29]. The compressor is a vital part of the fast-fill operation and is the main contributor to the CNG fueling station's operating cost through its power consumption as well as wear and tear [30]. The sizing of the station compressor and other station components is based on the expected inlet flow rate from the municipal supply line and the quantity of gas expected to be dispensed at the station [31]. Efficient operation of the compressor in a CNG fuelling station presents an opportunity for the reduction of operating costs. The savings that are realised can be passed on to consumers in the form of reduced price of $\mathrm{CNG}$ per unit of sale.

Energy efficiency of energy converting systems falls into four general categories of equipment efficiency, technology efficiency, performance efficiency and operation efficiency [32, 33, 34, 35]. CNG fuelling station operators, just like other commercial electricity consumers, must make careful consideration for all the four categories of energy efficiency in order to increase the economic performance of these installations [36, 37, 38, 39].

Research into the efficient operation of CNG fuelling stations has been greatly aided by the 
work of previous researchers. Kountz [40] modelled the fast-fill process based on the first law of thermodynamics for gas behaviour between a single reservoir and the on-board vehicle cylinder. Other researchers have expanded the modelling of the fast-fill CNG fueling station by considering the individual components of the station infrastucture and their interaction with the flowing gas. These include [41] and [42] whose work advanced the thermodynamic modelling of the fastfilling process. The research on further minimization of filling time has been studied by [43]. Using thermodynamic laws and mass balance, [44] studied the effects of initial conditions and ambient temperature on the filling of the vehicle on-board cylinder and achieving of the target pressure. The effects of the connecting pipe on the process of vehicle filling was also studied by [45]. Research on the complete filling of on-board vehicle cylinder through the development of dispenser algorithms for the fast-fill process has been conducted by [46] and [19]. Research has also been undertaken in relation to the thermodynamic behaviour of the reciprocating compressor in achieving different performance goals for the CNG fuelling station [47, 48, 49, 50]. Frick et al [51] studied the optimization of the distribution of CNG refuelling stations in Switzerland. The study applied cost benefit analysis to determine optimal location of new CNG fuelling stations among the existing petrol filling stations as well as existing CNG filling stations. CNG self-fuelling of vehicles and fuelling from homes has also been the subject of other research towards efficient delivery of the gas [16, 52] . The significant effect that domestic refuelling of CNG vehicles in consumer homes could have on the electric power infrastructure has been studied by [53]. Their study recognized limited infrastructure as a major technological barrier to the market penetration of CNG vehicles in the United States of America. This limitation was also shown to result in more consumers using domestic compressor units to fuel their vehicles, with potential effects on the electric grid network.

To the best of the authors' knowledge no research has been done on the implementation of electric load shifting for CNG fuelling stations in order to reduce their electricity costs charged at a time-of-use (TOU) tariff. Further, the simultaneous optimization of the operation of priority panel valves and the compressor for the purpose of achieving electric load scheduling for a CNG fuelling station has not been reported in the literature. This study presents an attempt to apply an optimized operation strategy to CNG fuelling stations in order to secure the benefits of demand response (DR) programs implemented by power utility operators through the time-of-use tariff for both the utility and the fuelling station, by means of shifting the stations electricity load out of high demand periods. This study also implements, evaluates and compares novel strategies for minimizing of the compressor switching frequency to mitigate wear and tear of compressor components caused by frequent on/off operation. The time-of-use tariff is implemented in order to encourage change in electricity usage by end users from the normal use pattern in response to change in electricity pricing based on time [54, 55, 56]. DR programs are implemented to cause intentional modifications of electricity consumption patterns by end-use customers, to alter the timing, demand level and total electricity consumption [57, 58, 59, 60]. The present work seeks to explore the implementation of a response to the time-of-use DR program for the CNG fuelling station by tracking the gas demand profile. An optimization strategy is used to alter the operation of the compressor in order to achieve a reduction in electricity costs thereby lowering operating costs of the station. Lowering of the operating costs of CNG fuelling stations can improve the attractiveness of $\mathrm{CNG}$ as a fuel through passing on of some of the accrued savings to consumers in 
the form of reduced prices on $\mathrm{CNG}$ per unit of sale. The proposed method achieves a breakthrough in scheduling the on/off switching of the compressor while considering CNG demand, the electricity TOU pricing and the protection of the compressor from damage caused by excessive on-off cycling. The proposed methods provide measures for optimal use of energy resources through optimization of energy processes in alternative fuels.

\section{Operation modelling and formulation}

The schematic diagram of a CNG fast-fill refuelling station unit with a cascade storage system is shown in Figure (1). The cascade storage tanks are supplied from the municipal gas supply line via the station compressor when it is turned on at switch $u$. The compressor switch is activated when pressure in the three reservoir tanks of the cascade storage falls to the lower limits [61]. The gas enters the storage tanks via a priority panel which is controlled to switch the incoming gas flow between the reservoir tanks by activating valves $u_{h p}$ for the high pressure tank, $u_{m p}$ for the medium pressure tank and $u_{l p}$ for the low pressure tank. Only one of the three valves is activated at a time. When the maximum pressure limits are reached, the compressor is switched off. Gas from the cascade storage tanks is supplied to vehicle tank via a dispenser with a dispensing algorithm that compensates for the variation in temperature to ensure the correct quantities are transferred to vehicle tanks [24]. Depending on vehicle tank pressure, the dispenser switches the gas supply between the three cascade storage tanks to sustain flow of gas above a minimum flow rate. Considering the metered gas demand from the three reservoirs for the cascade storage $m_{\text {ohp }}, m_{\text {omp }}$ and $m_{\text {olp }}$ for the high pressure reservoir, medium pressure reservoir and low pressure reservoir respectively, the proposed strategy seeks to optimally schedule the on/off switching of the compressor via switch $u$, as well as the priority panel valves $u_{h p}, u_{m p}$ and $u_{l p}$ in order to minimize the cost of power consumed by the compressor when electricity is purchased under a TOU tariff. The status of the compressor switch $u$ and the status of the priority panel valves $u_{h p}, u_{m p}$ and $u_{l p}$ are the control variables in the current problem.

\subsection{The objective function}

The objective is to minimize the cost of power consumed by the CNG fuelling station compressor within the limits of the cascade storage system over the control horizon [62]. The objective function is therefore expressed as

$$
J=\sum_{t=1}^{N} p_{c o} p_{e}(t) u(t) t_{s}
$$

where $p_{c o}$ is the power rating of the electric motor driving the compressor, $p_{e}(t)$ is the price of electricity per $\mathrm{kWh}$ in a sampling interval, $N$ is the total number of samples and $t_{s}$ is the sampling interval. The horizon in the present work is 24 hours, divided into a sampling interval of 4 minutes yielding a total number of samples $N=\frac{24 \times 60}{4}=360$. The sampling time of 4 minutes represents the average fuelling time of vehicles visiting a typical CNG fast-fill station [29]. [63].

\subsection{Constraints}

The problem in this study is subject to the following constraints. 


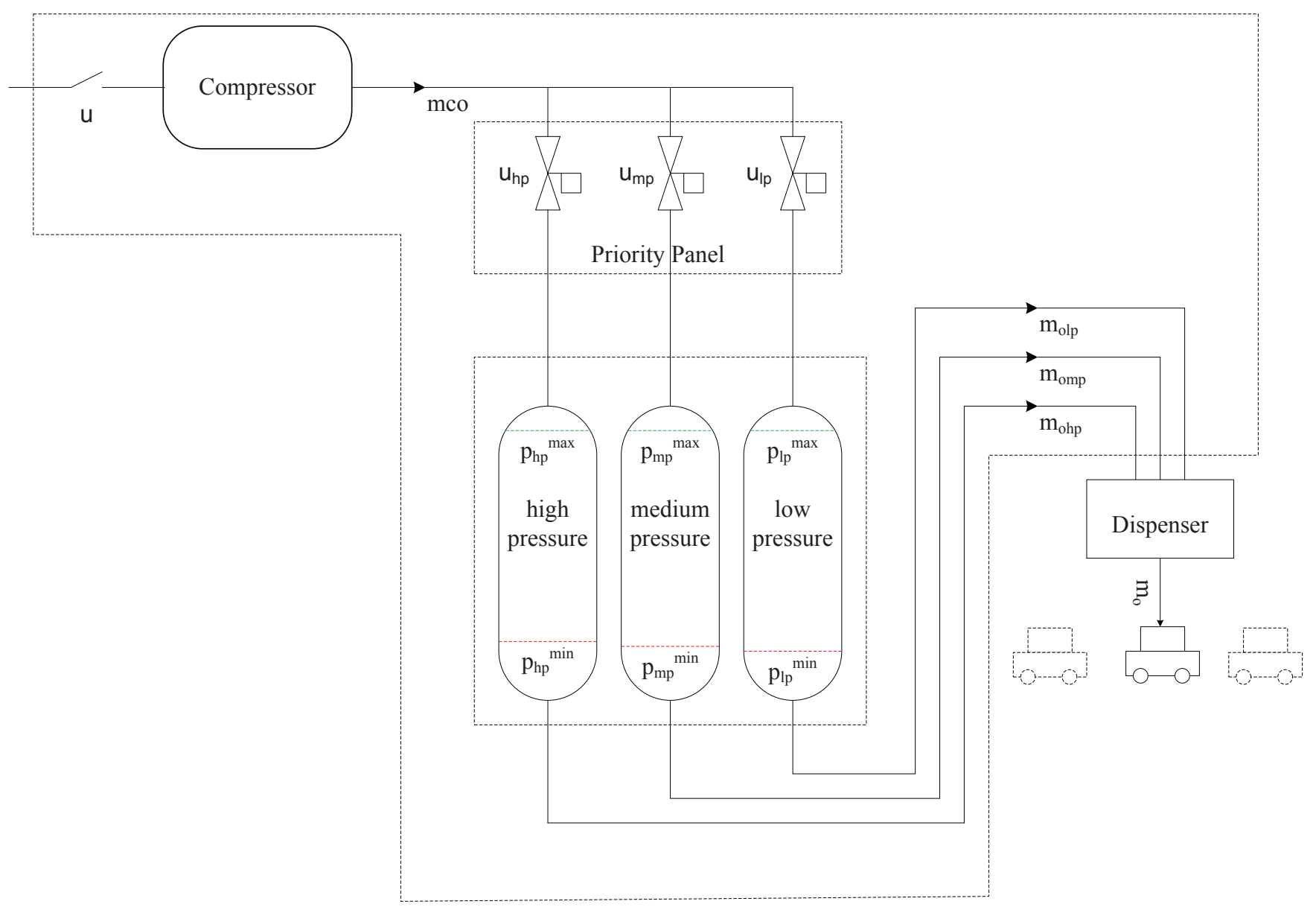

Figure 1: Schematic of a CNG fuelling station with a cascade storage system

\subsubsection{Reservoir capacity}

The mass of gas in the cascade storage high pressure, medium pressure and low pressure tanks in the $t$ th sampling interval is

$$
\begin{gathered}
m_{h p}(t)=m_{h p}(0)+\sum_{i=1}^{t-1} t_{s} \dot{m}_{c m p} u_{h p}(i)-\sum_{i=1}^{t-1} m_{\text {ohp }}(i) \\
m_{m p}(t)=m_{m p}(0)+\sum_{i=1}^{t-1} t_{s} \dot{m}_{c m p} u_{m p}(i)-\sum_{i=1}^{t-1} m_{\text {omp }}(i) \\
m_{l p}(t)=m_{l p}(0)+\sum_{i=1}^{t-1} t_{s} \dot{m}_{c m p} u_{l p}(i)-\sum_{i=1}^{t-1} m_{\text {olp }}(i)
\end{gathered}
$$

respectively, where $m_{\text {ohp }}, m_{\text {omp }}$ and $m_{\text {ohp }}$ are the values of mass dispensed in the $i$ th sampling instant from the high pressure, medium pressure and low pressure reservoirs respectively. $\dot{m}_{c m p}$ is 
the outlet mass flow rate of the compressor obtained using the equation (5) [29]

$$
\dot{m}_{c m p}=\rho_{s t d} \times Q_{s t d}=\left(\frac{M w_{g}}{M w_{a}}\right) \times \rho_{a, s t d} \times Q_{s t d}
$$

where $\rho_{\text {std }}$ is the density of the gas being compressed under standard conditions $\left(0^{\circ} \mathrm{C}\right.$ temperature and $10^{5}$ pascals pressure) ${ }^{2}, M w_{g}$ is the molecular weight of the gas, $M w_{a}$ the molecular weight of air, $\rho_{a, s t d}$ is the air density under standard conditions and $Q_{s t d}$ is the capacity of the compressor under standard conditions.

The mass in the tanks in equations (2), (3) and (4) must be sustained at values between the masses corresponding to the maximum pressure and minimum pressure for the three reservoirs respectively such that

$$
\begin{aligned}
& m_{h p}^{\min } \leq m_{h p}(0)+\sum_{i=1}^{t-1} t_{s} \dot{m}_{c m p} u_{h p}(i)-\sum_{i=1}^{t-1} m_{o h p}(i) \leq m_{h p}^{\max } \\
& m_{m p}^{\min } \leq m_{m p}(0)+\sum_{i=1}^{t-1} t_{s} \dot{m}_{c m p} u_{m p}(i)-\sum_{i=1}^{t-1} m_{o m p}(i) \leq m_{m p}^{\max } \\
& m_{l p}^{\min } \leq m_{l p}(0)+\sum_{i=1}^{t-1} t_{s} \dot{m}_{c m p} u_{l p}(i)-\sum_{i=1}^{t-1} m_{o l p}(i) \leq m_{l p}^{\max }
\end{aligned}
$$

where $m_{h p}^{\min }, m_{m p}^{\min }$ and $m_{m p}^{\min }$ are the values of minimum quantity of gas for the high pressure, medium pressure and low pressure reservoirs respectively, while $m_{h p}^{\max }, m_{m p}^{\max }$ and $m_{l p}^{\max }$ are the values for the maximum quantity of gas for the high pressure, medium pressure and low pressure reservoirs respectively. These quantity limits can be obtained from the relationship between gas properties and the system rated pressure limits in the equation of state

$$
p V=z n R T
$$

where $p$ is the pressure, $V$ the volume, $T$ the absolute temperature, $R$ is the universal gas constant, $z$ the compressibility factor and $n$ is the quantity of gas in moles [64, 65].

$$
n=\frac{m}{M}=\frac{p V}{z R T}
$$

where $m$ is the mass of the gas and $M$ is the molar mass of the gas. Equation (10) can be used to determine the upper and lower limits of the mass content of the reservoir tanks in the cascade storage of the CNG refuelling station. In the present work we consider the effect of the ambient temperature on the cascade storage by assuming that the cascade storage reservoirs normalize to the ambient temperature during charging and discharging. The maximum and minimum temperatures for the control horizon are taken as the day's highest and lowest temperatures respectively. When the ambient temperature rises, the pressure in the reservoirs will rise. If the upper pressure

\footnotetext{
$2^{2}$ https://goldbook.iupac.org/html/S/S05910.html
} 
limit is breached, the safety release valve will open to expel excess gas into the atmosphere. It is therefore necessary to calculate and set the upper limit of mass that can be held in the reservoir at the highest temperature in the control horizon. Similarly, the lower limit of the reservoirs must be calculated and set at the lowest temperature to prevent the pressure of gas held in storage from falling below the lower limit when ambient temperature falls, causing a pressure drop. The limits are therefore calculated as

$$
\begin{array}{ll}
m_{h p}^{\max }=\frac{M V p_{h p}^{\max }}{z R T_{\text {max }}} & m_{h p}^{\text {min }}=\frac{M V p_{h p}^{\text {mim }}}{z R T_{\text {min }}} \\
m_{m p}^{\text {max }}=\frac{M V p_{m p}^{\max }}{z R T_{\text {max }}} & m_{m p}^{\text {min }}=\frac{M V p_{m p}^{\text {min }}}{z R T_{\text {min }}} \\
m_{l p}^{\max }=\frac{M V p_{l p}^{\max }}{z R T_{\text {max }}} & m_{l p}^{\min }=\frac{M V p_{l p}^{\min }}{z R T_{\text {min }}}
\end{array}
$$

where $p_{h p}^{\max }, p_{m p}^{\max }$ and $p_{l p}^{\max }$ are the maximum pressure limits for the respective reservoirs, $p_{h p}^{\min }, p_{m p}^{\min }$ and $p_{l p}^{\min }$ are the minimum pressure limits for the respective reservoirs and $T_{\max }$ and $T_{\min }$ are the maximum and minimum ambient temperatures in the control horizon respectively.

\subsubsection{Switching combinations}

During operation, whenever the compressor switch $u$ is turned on, only one of the valves $u_{h p}$, $u_{m p}$ and $u_{l p}$ can be on [29, 66] and all the valves must be off whenever the compressor is off such that

$$
u_{h p}+u_{m p}+u_{l p}-u=0
$$

\subsection{Compressor switching frequency}

Frequent on/off switching increases mechanical stress induced in the compressor components, which causes an increase in wear and tear [67] and therefore increases the maintenance costs, while reducing the life of the compressor [68]. Transient start-up and shut-down states of the compressor have been shown to induce the highest stresses in different compressor components [69]. Additionally, there are vibrations resulting from torsional oscillations caused by loading changes throughout the compressor shaft, seals and coupled mechanisms as the motor pulls the load towards stabilization which cause further wear and tear [70]. It is therefore desirable that after the compressor is turned on, it should be kept running for as long as possible, meaning it should operate in wide on or off state bands [71]. This is the motivation of presenting switch frequency minimization in our work. It would be more accurate to directly minimize the actual wear and tear caused by switching in the model instead of minimizing the switching numbers. However, there is no accurate model that captures the relationship between the switching actions and the wear and tear. Because of lack of such models in literature, the approach by many researchers is to penalise the occurrence of transient states when optimizing operation of rotary machine systems such as compressors [62, 67, 72]. This is still a valid approach because it is a known prior that the wear and tear increases with the increase of switching frequency. 
In this study, we aim to achieve the least number of switching instances that does not raise the cost of electricity when compared to the cost achieved during optimization without penalizing of switching frequency. We implement two methods of achieving a minimum number of switching instances of the compressor. The first method is the Pretoria method proposed by [73] and used subsequently in optimization of the performance of water pumping systems [63, 74]. The Pretoria method makes use of an auxiliary variable $s(t)$ which assumes a value of 1 whenever a switch start-up occurs. The auxiliary variable is then minimized in the objective function (36) which is then expressed as

$$
J=\xi \sum_{t=1}^{N} p_{c o} p_{e}(t) u(t) t_{s}+(1-\xi) \sum_{t=1}^{N} s(t)
$$

where $\xi$ is a weighting factor. Additional to the constraints in 2.2.1 and 2.2.2, the problem is subject to further constraints arising from the use of the auxiliary variable. The constraints are

$$
\begin{gathered}
u(1)-s(1) \leq 0 \\
u(t)-u(t-i)-s(t) \leq 0
\end{gathered}
$$

where the inequality $(16)$ initializes the auxiliary variable as the initial status of $u$ while the inequality (17) favours the control that involves less switching instances [75]. The Pretoria method was shown to be superior to a method proposed by [63] that used constraints to restrict the number of on/off instances for the switch. The constraint method was determined to run at risk of infeasibility in certain control conditions.

We propose a second approach we call the non-linear objective function method which is describe in section (2.6)

\subsection{Boundaries}

The condition of the switch $u$ and the valves $u_{h p}, u_{m p}$ and $u_{l p}$ as well as the auxiliary variable is binary such that

$$
u(t), u_{h p}(t), u_{m p}(t), u_{l p}(t), s(t) \in\{0,1\} \quad(1 \leq t \leq N)
$$

\subsection{Algorithm}

The generalized optimization problem in the present study is to minimize $f^{T} X$ subject to equality constraints $\left(A_{e q} X=b_{e q}\right)$, inequality constraints $(A X \leq b)$ and the upper and lower boundaries of the control variables $\left(L_{B} \leq X \leq U_{B}\right)$ [76]. The control variables $u(t), u_{h p}(t), u_{m p}(t), u_{l p}(t)$ and $s(j)$ are contained in vector $X$, while $A$ and $A_{e q}$ are matrices. $b, L_{B}$ and $U_{B}$ are vectors represented as

$$
X=\left[u(1) \cdots u(N) \quad u_{h p}(1) \cdots u_{h p}(N) \quad u_{m p}(1) \cdots u_{m p}(N) \quad u_{l p}(1) \cdots u_{l p}(N) \quad s(1) \cdots s(N)\right]_{5 N \times 1}^{T}
$$

and the objective function as

$$
f^{T}=\left[\begin{array}{ccccc}
\xi p_{c o} t_{s} p_{e}(1) \cdots \xi p_{c o} t_{s} p_{e}(N) & 0 \cdots 0 & 0 \cdots 0 & 0 \cdots 0 & (1-\xi) \cdots(1-\xi)
\end{array}\right]_{1 \times 5 N}
$$


from the inequality constraints $(6),(7),(8),(16)$ and $(17)$, if we denote

$$
\begin{gathered}
A_{c}=\left[\begin{array}{cccc}
-t_{s} \dot{m}_{c m p} & 0 & \cdots & 0 \\
-t_{s} \dot{m}_{c m p} & -t_{s} \dot{m}_{c m p} & \cdots & 0 \\
\vdots & \vdots & \ddots & \vdots \\
-t_{s} \dot{m}_{c m p} & -t_{s} \dot{m}_{c m p} & \cdots & -t_{s} \dot{m}_{c m p}
\end{array}\right]_{N \times N} \\
A_{u}=\left[\begin{array}{ccccc}
1 & 0 & 0 & \cdots & 0 \\
-1 & 1 & 0 & \cdots & 0 \\
0 & -1 & 1 & \cdots & 0 \\
\vdots & \vdots & \vdots & \ddots & \vdots \\
0 & 0 & \cdots & -1 & 1
\end{array}\right]_{N \times N}
\end{gathered}
$$

then

$$
\begin{aligned}
& A_{1}=\left[\begin{array}{lllll}
0 & A_{c} & 0 & 0 & 0
\end{array}\right]_{N \times 5 N} \\
& A_{2}=\left[\begin{array}{lllll}
0 & 0 & A_{c} & 0 & 0
\end{array}\right]_{N \times 5 N} \\
& A_{3}=\left[\begin{array}{lllll}
0 & 0 & 0 & A_{c} & 0
\end{array}\right]_{N \times 5 N} \\
& A_{4}=\left[\begin{array}{lllll}
A_{u} & 0 & 0 & 0 & -I
\end{array}\right]_{N \times 5 N} \\
& b_{1}=\left[\begin{array}{c}
m_{h p}(0)-m_{h p}^{\min }-m_{o h p}(1) \\
m_{h p}(0)-m_{h p}^{\min }-\left(m_{\text {ohp }}(1)+m_{o h p}(2)\right) \\
\vdots \\
m_{h p}(0)-m_{h p}^{\min }-\left(m_{\text {ohp }}(1)+m_{\text {ohp }}(2)+\cdots+m_{\text {ohp }}(N)\right)
\end{array}\right]_{N \times 1} \\
& b_{2}=\left[\begin{array}{c}
m_{h p}^{\max }-m_{h p}(0)+m_{\text {ohp }}(1) \\
m_{h p}^{\max }-m_{h p}(0)+\left(m_{\text {ohp }}(1)+m_{\text {ohp }}(2)\right) \\
\vdots \\
m_{h p}^{\max }-m_{h p}(0)+\left(m_{\text {ohp }}(1)+m_{\text {ohp }}(2)+\cdots+m_{\text {ohp }}(N)\right)
\end{array}\right]_{N \times 1}
\end{aligned}
$$




$$
\begin{aligned}
& b_{3}=\left[\begin{array}{c}
m_{m p}(0)-m_{m p}^{\min }-m_{\text {omp }}(1) \\
m_{m p}(0)-m_{m p}^{\min }-\left(m_{\text {omp }}(1)+m_{\text {omp }}(2)\right) \\
\vdots \\
m_{m p}(0)-m_{m p}^{\min }-\left(m_{\text {omp }}(1)+m_{\text {omp }}(2)+\cdots+m_{\text {omp }}(N)\right)
\end{array}\right]_{N \times 1} \\
& b_{4}=\left[\begin{array}{c}
m_{m p}^{m a x}-m_{m p}(0)+m_{o m p}(1) \\
m_{m p}^{m a x}-m_{m p}(0)+\left(m_{o m p}(1)+m_{o m p}(2)\right) \\
\vdots \\
m_{m p}^{\max }-m_{m p}(0)+\left(m_{\text {omp }}(1)+m_{\text {omp }}(2)+\cdots+m_{\text {omp }}(N)\right)
\end{array}\right]_{N \times 1} \\
& b_{5}=\left[\begin{array}{c}
m_{l p}(0)-m_{l p}^{\min }-m_{o l p}(1) \\
m_{l p}(0)-m_{l p}^{\min }-\left(m_{o l p}(1)+m_{o l p}(2)\right) \\
\vdots \\
m_{l p}(0)-m_{l p}^{\min }-\left(m_{\text {olp }}(1)+m_{\text {olp }}(2)+\cdots+m_{\text {olp }}(N)\right)
\end{array}\right]_{N \times 1} \\
& b_{6}=\left[\begin{array}{c}
m_{l p}^{\max }-m_{l p}(0)+m_{\text {olp }}(1) \\
m_{l p}^{\max }-m_{l p}(0)+\left(m_{\text {olp }}(1)+m_{\text {olp }}(2)\right) \\
\vdots \\
m_{l p}^{\max }-m_{l p}(0)+\left(m_{\text {olp }}(1)+m_{\text {olp }}(2)+\cdots+m_{\text {olp }}(N)\right)
\end{array}\right]_{N \times 1} \\
& b_{7}=[0]_{N \times 1}
\end{aligned}
$$

then the linear inequality constraints become

$$
A=\left[\begin{array}{c}
A_{1} \\
-A_{1} \\
A_{2} \\
-A_{2} \\
A_{3} \\
-A_{3} \\
A_{4}
\end{array}\right]_{7 N \times 5 N} \quad b=\left[\begin{array}{c}
b_{1} \\
b_{2} \\
b_{3} \\
b_{4} \\
b_{5} \\
b_{6} \\
b_{7}
\end{array}\right]_{7 N \times 1}
$$

for the equality constraint (14) we denote

$$
A_{e q}=\left[\begin{array}{lllll}
-I & I & I & I & 0
\end{array}\right]_{N \times 5 N} \quad b_{e q}=[0]_{N \times 1}
$$

This binary linear optimization problem is solved using the MATLAB Solving Constraint Integer Programs (SCIP) solver in the OPTI toolbox interface. 


\subsection{The non-linear objective function method to minimize compressor switching instances}

In this method, we introduce the quadratic element $\sum(u(t+1)-u(t))^{2}$ to the objective function. The element minimizes the rate of change of status of the switch over the control horizon so as to achieve longer operating bands in both on and off states. The control variables are $u, u_{h p}, u_{m p}$ and $u_{l p}$ and the objective function (36) using this method becomes

$$
J=\psi \sum_{t=1}^{N} p_{c o} p_{e}(t) u(t) t_{s}+(1-\psi) \sum_{t=1}^{N-1}(u(t+1)-u(t))^{2}
$$

where $\psi$ is a weighting factor. The constraints in this approach remain as in section 2.2.1) and (2.2.2). The optimization problem can be written in the standard form

$$
x=\left[u(1) \cdots u(N) \quad u_{h p}(1) \cdots u_{h p}(N) \quad u_{m p}(1) \cdots u_{m p}(N) \quad u_{l p}(1) \cdots u_{l p}(N)\right]_{4 N \times 1}^{T}
$$

and in the general OPTI toolbox solver algorithm the objective function is formulated as

$$
\min _{x} f^{T} x \text { subject to }=\left\{\begin{array}{l}
A \cdot x \leq b \\
A_{e q} \cdot x \leq b_{e q} \\
l_{b} \leq x \leq U_{b} \\
x \in\{0,1\}
\end{array}\right.
$$

from the linear inequality constraints $(6),(7),(8)$, we can denote

$$
\begin{aligned}
& A_{1}^{\prime}=\left[\begin{array}{llll}
0 & A_{c} & 0 & 0
\end{array}\right]_{N \times 5 N} \\
& A_{2}^{\prime}=\left[\begin{array}{llll}
0 & 0 & A_{c} & 0
\end{array}\right]_{N \times 5 N} \\
& A_{3}^{\prime}=\left[\begin{array}{llll}
0 & 0 & 0 & A_{c}
\end{array}\right]_{N \times 5 N}
\end{aligned}
$$

then the linear inequality constraints become

$$
A^{\prime}=\left[\begin{array}{c}
A_{1}^{\prime} \\
-A_{1}^{\prime} \\
A_{2}^{\prime} \\
-A_{2}^{\prime} \\
A_{3}^{\prime} \\
-A_{3}^{\prime}
\end{array}\right]_{6 N \times 4 N} \quad b^{\prime}=\left[\begin{array}{l}
b_{1} \\
b_{2} \\
b_{3} \\
b_{4} \\
b_{5} \\
b_{6}
\end{array}\right]_{6 N \times 1}
$$

for the equality constraint (14) we can denote

$$
A_{e q}^{\prime}=[-I ;-I ;-I ;-I]_{N \times 4 N} \quad b_{e q}^{\prime}=[0]_{N \times 1}
$$

This non-linear optimization problem is also solved using the MatLab SCIP solver in the OPTI toolbox interface. 


\subsection{Consideration for terminal constraints}

It is desirable that the quantity of gas available at the end of the control horizon be similar to the initial quantity of gas in the cascade storage to ensure that the initial conditions are repeated for the next control period because open loop strategies do not guarantee proper operation in the subsequent control periods if initial conditions are not the same [74, 77]. From Equations 2, 3] and 4. the mass of gas in the three tanks of the cascade storage at the end of the control horizon can be set as;

$$
\begin{gathered}
m_{h p}(N)=m_{h p}(0)=m_{h p}(0)+\sum_{i=1}^{N} t_{s} \dot{m}_{c m p} u_{h p}(i)-\sum_{i=1}^{N} m_{\text {ohp }}(i) \\
m_{m p}(N)=m_{m p}(0)=m_{m p}(0)+\sum_{i=1}^{N} t_{s} \dot{m}_{c m p} u_{m p}(i)-\sum_{i=1}^{N} m_{\text {omp }}(i) \\
m_{l p}(N)=m_{l p}(0)=m_{l p}(0)+\sum_{i=1}^{N} t_{s} \dot{m}_{c m p} u_{l p}(i)-\sum_{i=1}^{N} m_{\text {olp }}(i)
\end{gathered}
$$

It was deemed necessary to implement the terminal constraints as soft constraints, considering a fixed mass of gas flows into the cascade storage when the compressor is switched on in a sampling interval. This may not allow the solution to be automatically feasible with a hard constraint. The softened terminal constraint is a restriction of the final mass of gas in the cascade storage to within $90 \%$ of the initial mass in a control period. Therefore from equations (44), (45) and (46)

$$
\begin{gathered}
0.9 \times m_{h p}(0) \leq m_{h p}(0)+\sum_{i=1}^{N} t_{s} \dot{m}_{c m p} u_{h p}(i)-\sum_{i=1}^{N} m_{\text {ohp }}(i) \leq m_{h p}(0) \\
0.9 \times m_{m p}(0) \leq m_{m p}(0)+\sum_{i=1}^{N} t_{s} \dot{m}_{c m p} u_{m p}(i)-\sum_{i=1}^{N} m_{\text {omp }}(i) \leq m_{m p}(0) \\
0.9 \times m_{l p}(0) \leq m_{l p}(0)+\sum_{i=1}^{N} t_{s} \dot{m}_{c m p} u_{l p}(i)-\sum_{i=1}^{N} m_{\text {olp }}(i) \leq m_{l p}(0)
\end{gathered}
$$

\section{Case data}

\subsection{The CNG fuelling station}

A CNG fuelling station in Johannesburg, South Africa is used as a case for the present study. The fuelling station has two dispensers with four refuelling nozzles and one reciprocating compressor. The fuelling station is supplied with gas from the utility company contracted by Johannesburg municipality to operate the municipality's gas pipeline. Table (1) shows the specifications of the CNG fuelling station unit. Under baseline operation, the low pressure limits of the cascade storage trigger the switching on of the compressor while the high pressure limits trigger the switching off. 
Table 1: CNG fuelling station data

\begin{tabular}{ll}
\hline Specification & value \\
\hline High pressure reservoir capacity & $2000 \mathrm{~L}$ \\
Medium pressure reservoir capacity & $2000 \mathrm{~L}$ \\
Low pressure reservoir capacity & $2000 \mathrm{~L}$ \\
Maximum Pressure for all reservoir levels & $252 \mathrm{bar}$ \\
High pressure reservoir minimum pressure & $210 \mathrm{bar}$ \\
Medium pressure reservoir minimum pressure & $150 \mathrm{bar}$ \\
Low pressure reservoir minimum pressure & $75 \mathrm{bar}$ \\
Priority panel & $3 \mathrm{lines}$ \\
Compressor capacity & $900 \mathrm{Nm} / \mathrm{hr}$ \\
Compressor motor rating & $132 \mathrm{~kW}$ \\
\hline
\end{tabular}

\subsection{Time-of-use electricity tarrif}

The time-of-use (TOU) electricity tariff is used in the electricity power industry so that retail electricity pricing is such that it reflects changes in the wholesale electricity market due to electricity demand [78]. It may vary by time of day, by day of the week and by seasons [79, 36]. South Africa electric utility company, Eskom's tarrif f $^{3}$ for businesses named the TOU Miniflex is applicable to the CNG station in this study. The tariff is implemented at two levels; seasonal as well as time of day. Seasonal pricing is divided into high demand season in the winter months of June to August while the rest of the year is priced as low demand season, September to May. Further, the peak, standard and off-peak times in the day differ in the two seasons such that

$$
\begin{gathered}
p_{\text {eHD }}(t)= \begin{cases}p_{\text {offpeak }}=0.5157 \mathrm{R} / \mathrm{kWh} & \text { if } t \in[0,6] \cup[22,24] \\
p_{\text {standard }}=0.9446 \mathrm{R} / \mathrm{kWh} & \text { if } t \in[9,17] \cup[19,22] \\
p_{\text {peak }}=3.1047 \mathrm{R} / \mathrm{kWh} & \text { if } t \in[6,9] \cup[17,19]\end{cases} \\
p_{\text {eLD }}(t)= \begin{cases}p_{\text {offpeak }}=0.4472 \mathrm{R} / \mathrm{kWh} & \text { if } t \in[0,6] \cup[22,24] \\
p_{\text {standard }}=0.7016 \mathrm{R} / \mathrm{kWh} & \text { if } t \in[6,7] \cup[10,18] \cup[20,22] \\
p_{\text {peak }}=1.0167 \mathrm{R} / \mathrm{kWh} & \text { if } t \in[7,10] \cup[18,20]\end{cases}
\end{gathered}
$$

where $p_{e H D}$ and $p_{e L D}$ is the price during high demand season and low demand season respectively, $p_{\text {off peak }}$ is the off-peak price, $p_{\text {standard }}$ the price at standard time and $p_{\text {peak }}$ the price at peak time, $R$ is the South African Rand and $t$ is the time of the day in hours. The tariff also has other charge components that are not considered in this study as they are constant [74].

\subsection{Gas demand}

The mass of gas flowing from the three CNG reservoir storage levels measured at the dispenser results in a gas demand profile shown in Figure (2) for two days; one in the high demand electricity

\footnotetext{
$\sqrt[3]{\text { http://eskom.co.za/tariffs }}$
} 


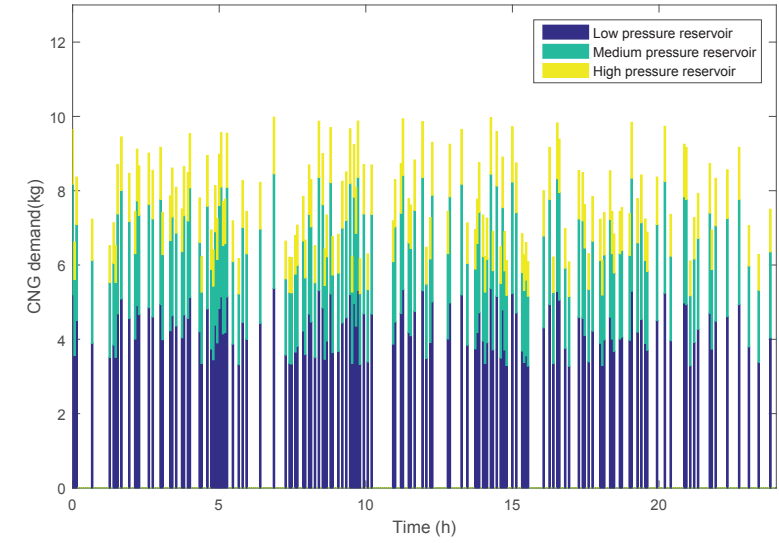

(a) High electricity demand season(143 vehicles)

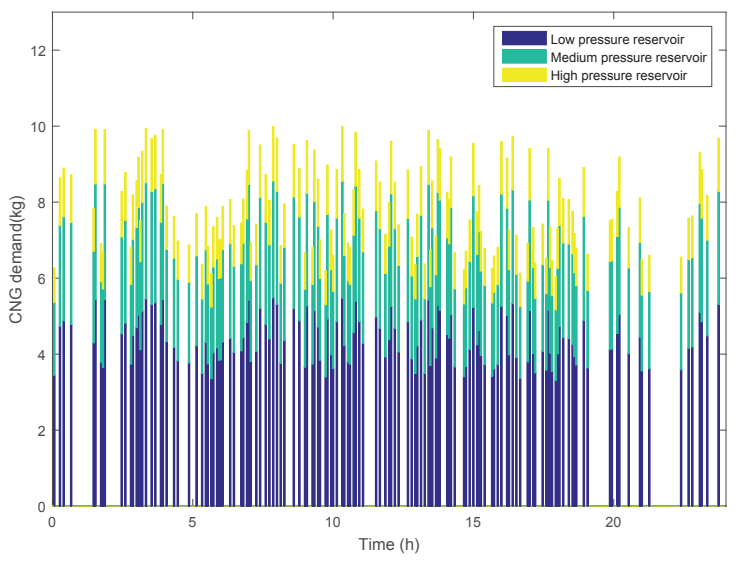

(b) Low electricity demand season(146 vehicles)

Figure 2: CNG demand from the three cascade storage levels for single day in each electricity pricing season

pricing season and the second in the low demand electricity pricing season. The dispenser uses mass flow measurements from each reservoir to make decision on valve sequencing when filling the vehicle tank and determine sale quantities with an operation log recording system performance [80]. The recorded mass of gas flowing from each reservoir for the two days in the two electricity pricing seasons bear similarities. From the profiles, there is an increase in gas demand in the early morning hours upto 10:00, due to motorists fuelling before beginning their journeys. There is also increased gas demand in the afternoon from 14:00 due to motorists refuelling in preparation for the evening rush hour. CNG powered vehicles are used mainly by courier fleet clients, security fleet clients and public service vehicles. Demand of gas tends to increase before and during people movement rush hours due to the public service transportation needs. Increased late afternoon and evening fuelling activity is also as a result of motorists who fuel prior to travelling the next day.

\section{Results and discussion}

\subsection{High demand electricity pricing season}

\subsubsection{Optimization without consideration for compressor switching frequency}

Figure (3) shows the system behaviour when optimized without taking into consideration the switching frequency of the compressor in the objective function. Before the end of the off-peak electricity pricing at 06:00, the activity of the compressor increases in order to fill up the three reservoirs and therefore reduce compressor action in the peak electricity pricing band between 06:00 and 09:00. The method successfully avoids turning on the compressor in the peak electricity pricing time band thereby saving energy cost. Since the compressor stays off during the morning peak electricity pricing period, the compressor has to be switched on to satisfy gas demand for the standard electricity pricing period. The mass of gas in the reservoir is maintained at a high level before the beginning of the second electricity peak pricing period, to reduce the activity of the compressor in the undesirable time band between, 17:00 and 19:00. The approach also succeeds in preventing compressor activity in this second peak electricity pricing period, further 
reducing the cost of electricity consumed for the day. The compressor is turned on minimally in the following standard electricity pricing band between 19:00 and 22:00 to enable meet gas demand before the onset of the off-peak electricity pricing period when the compressor can supply gas to meet the rest of the day's demand. By operating the filling of each reservoir independently, the minimum electricity cost is achieved through ensuring the compressor runs only when there is predicted demand on an individual reservoir. From a baseline electricity cost of R432.59 for the day, a reduction of electricity cost to R148.90 is realised. Although this optimization regime minimizes the compressor action during the peak periods of electricity pricing thereby reducing electricity cost, the number of on/off switching of the compressor is too high at 14 and increases the probability of failure through fatigue to the compressor components which will results in an increase in maintenance cost [68, 81].

\subsubsection{Optimization while considering compressor switching frequency}

Figure (4) and Figure (5) show the behaviour of the system when the switching frequency of the compressor is minimized using the Pretoria method with a weighting factor $\xi=0.01$ and the non-linear objective function method with a weighting factor $\psi=0.9$ respectively. These weighting factors were chosen to achieve the minimum number of switching instances without increasing the cost of electricity when compared to optimal operation without switching minimization. Both the Pretoria method and the non-linear objective function method are able to reduce the number of compressor turn-on instances to four when compared to the 14 instances in the optimized system without consideration for compressor switching frequency as can be seen in Figure 4a and Figure (5a). Compressor on-state is successfully avoided during the morning peak electricity pricing period between 06:00 and 09:00 by performing a reservoir refill just before the end of the off peak period at 06:00, prior to the onset of the peak electricity pricing period for both methods. Two compressor on-states occur in the standard electricity pricing time between 09:00 and 17:00 driven by the mid morning gas demand as well as the filling of the reservoirs just before the second peak electricity pricing period at 17:00. Both approaches are able to successfully prevent compressor activity in the second peak pricing period of the day and the subsequent standard electricity pricing period by predicting the demand in this time to ensure the compressor comes on only after the end of the standard electricity pricing period at 22:00.

The reduction in switching times for both the non-linear objective function method and the Pretoria method is achieved through synchronizing the utilization of the compressor to fill up each of the three reservoirs whenever an on-state occurs. Figure (5b) and Figure (4b) show how valve action is coordinated in the priority panel, to achieve gas levels in each of the reservoir that can sustain demand until the next synchronized need for a refill for the three reservoirs. The effects of the coordination on mass of gas in storage can be seen in the respective mass of gas in reservoir graphs on Figure (4c) and Figure (5c). The reduction in switching occurrences represents a 71.4\% reduction in the number of on/off actions that the compressor has to perform in comparison to optimization without consideration for the switching frequency. Fewer on/of instances mean a reduced probability of compressor component failure due to a high frequency of switching [82, 83]. This also reduces the maintenance costs of the CNG fuelling station unit for which the compressor is an critical component [15, 84].

A comparison of the three approaches to minimization of energy cost in the high demand 


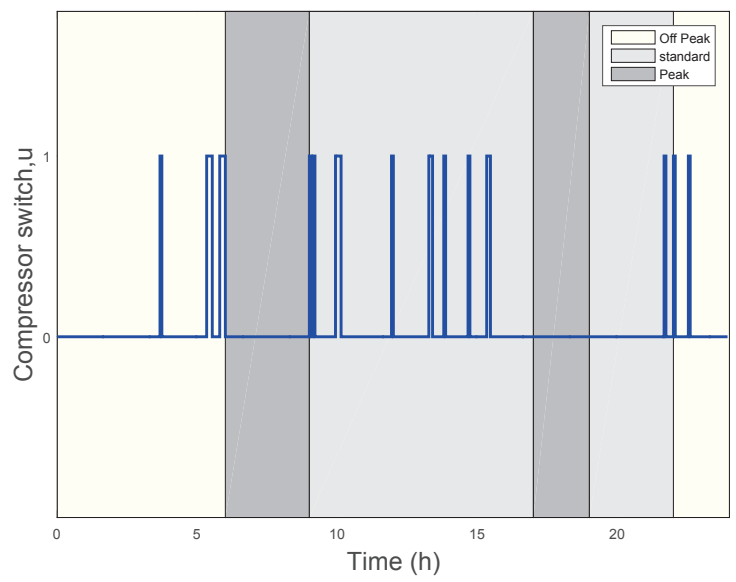

(a) Compressor switch action

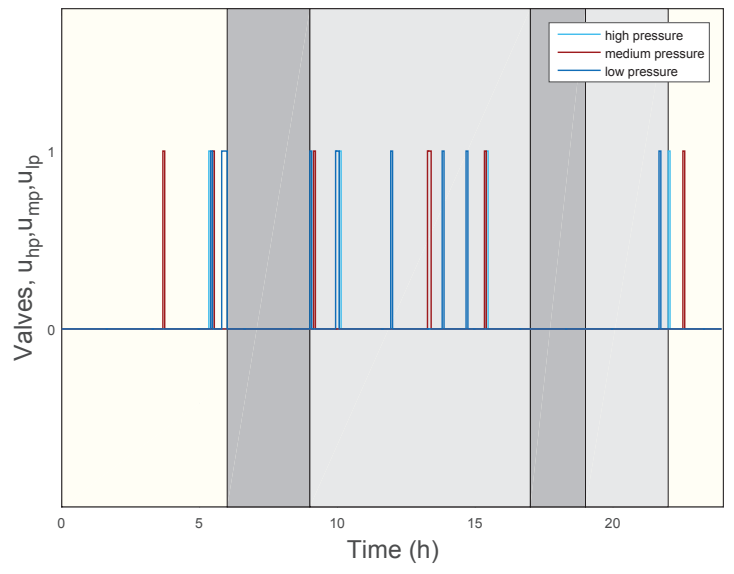

(b) Valve action

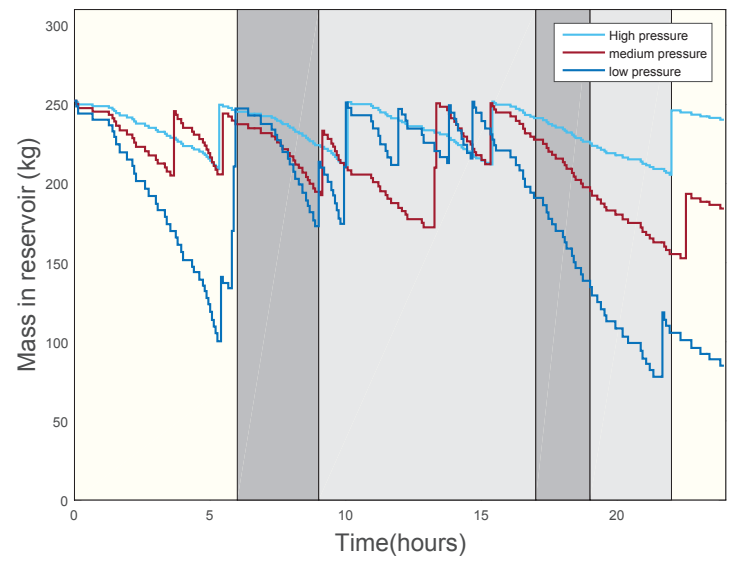

(c) Mass of gas in reservoir

Figure 3: System behaviour in the high demand electricity pricing season without consideration for compressor switching frequency 


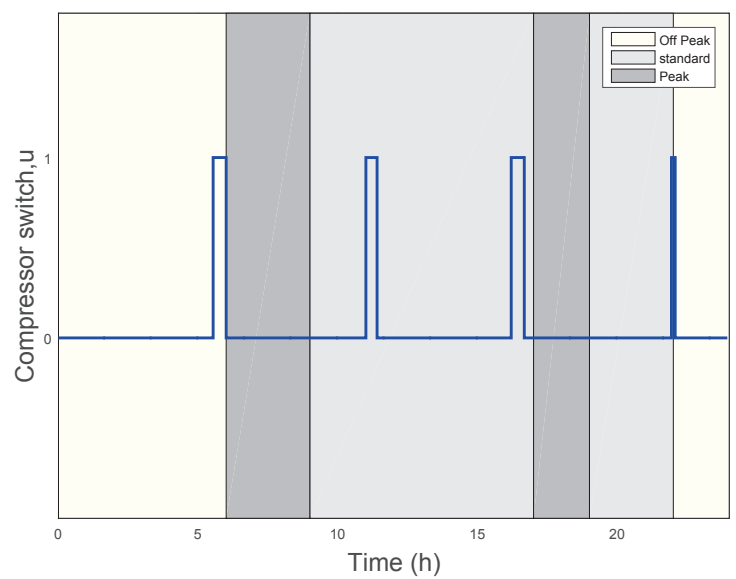

(a) Compressor switch action

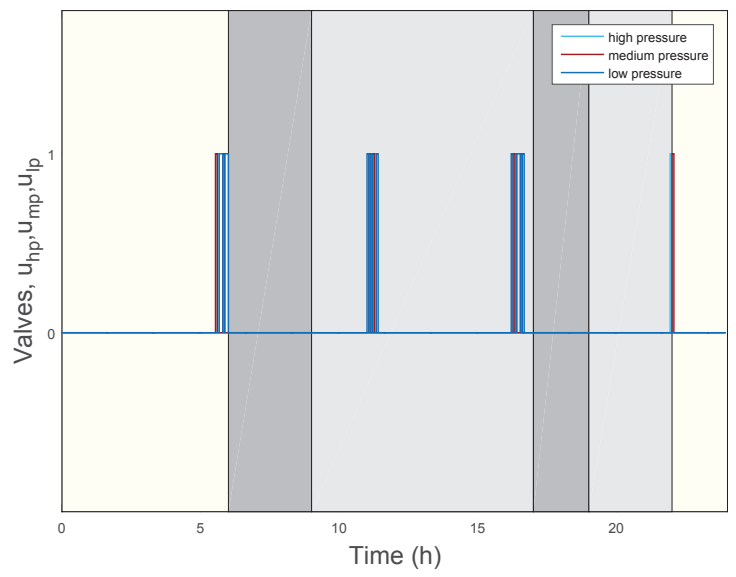

(b) Valve action

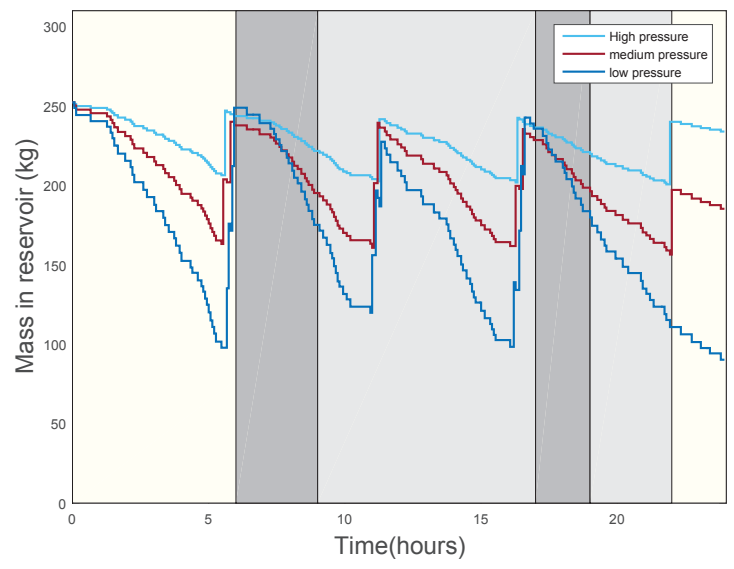

(c) Mass of gas in reservoir

Figure 4: System behaviour in the high demand electricity pricing season using the Pretoria method of minimizing compressor switching 


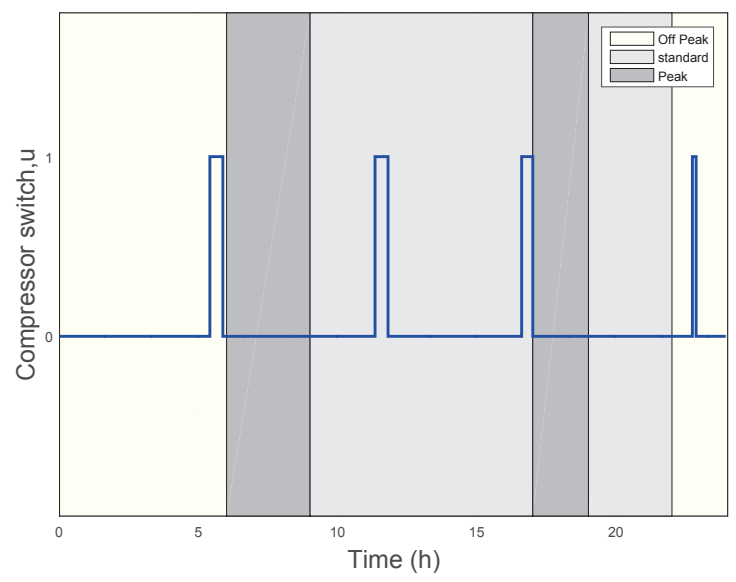

(a) Compressor switch action

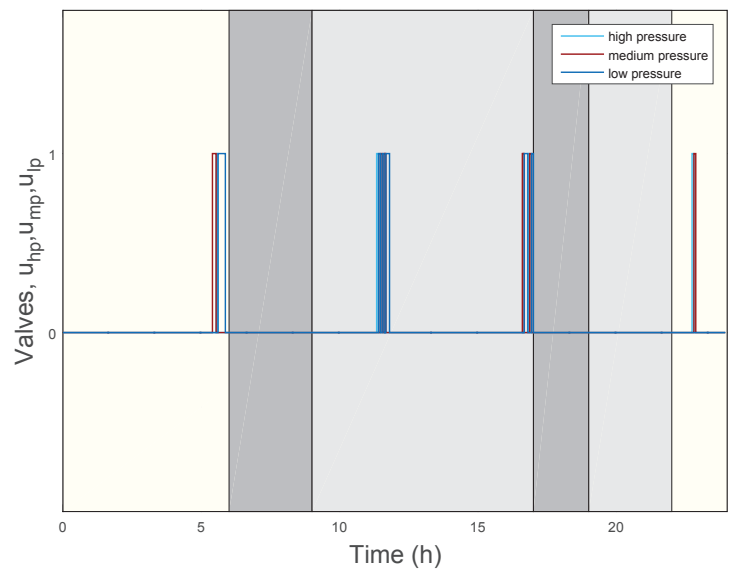

(b) Valve action

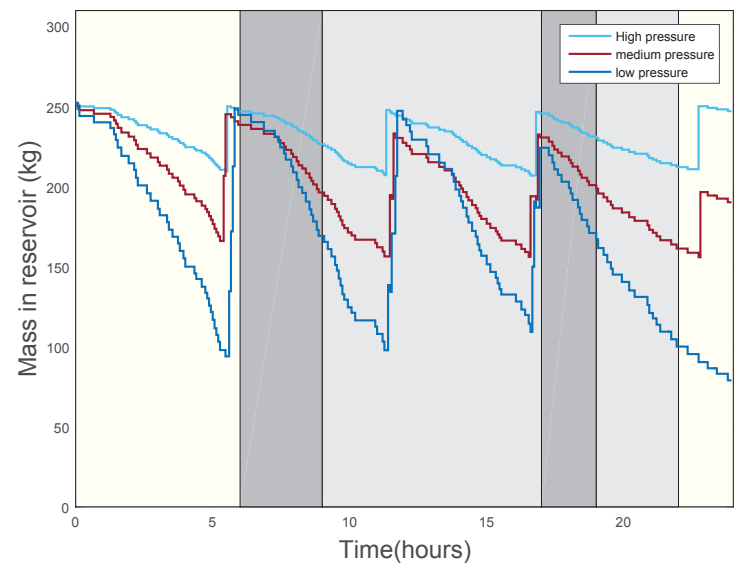

(c) Mass of gas in reservoir

Figure 5: System behaviour in the high demand electricity pricing season using the non-linear objective function method of minimizing compressor switching 
electricity pricing season is shown in Table (2). From a baseline cost of power of R432.59, all the methods studied in the present work are able to reduce the cost to R148.90. The three strategies yield the same costs of electricity because they deliver the same effective compressor operation time in each electricity pricing period with variations only occurring in the exact time when the compressor-on state occurs and the length of time the compressor stays on under each strategy. Although the optimization strategy without switching control yields similar electricity cost savings as with the other two and with less computing time, the number of switching instances is too high and exposes the CNG fuelling station unit to higher probabilities of failure. The non-linear objective function method is demonstrably superior to the Pretoria method, given that it yields equal cost savings in a shorter computing time which corresponds to lower computing costs [85]. The optimization of the operation of a CNG fuelling station compressor and priority panel using the non-linear objective function method of compressor cost minimization is concluded as the superior approach to achieve the objective of electricity cost reduction in a TOU tariff electricity pricing regime as well as compressor care.

Table 2: Comparison of performance for the control strategies

\begin{tabular}{llll}
\hline & Electricity cost (Rands) & $\begin{array}{l}\text { Switching } \\
\text { instances }\end{array}$ & Computing time (seconds) \\
\hline $\begin{array}{l}\text { Optimal operation without } \\
\text { switching minimization }\end{array}$ & 148.90 & 16 & 0.5 \\
\hline $\begin{array}{l}\text { Optimal operation with Preto- } \\
\text { ria switching minimization }\end{array}$ & 148.90 & 4 & 71 \\
\hline $\begin{array}{l}\text { Optimal operation with } \\
\text { non-linear Objective function } \\
\text { switching minimization }\end{array}$ & 148.90 & 4 & 21 \\
\hline
\end{tabular}

\subsection{Low demand electricity pricing season}

When applied to optimize operation of the CNG station considering the low electricity demand pricing season, the non-linear objective function method which has been determined to be the superior approach to the current problem in Section (4.1) results in the system behaviour shown in Figure (6). To avoid compressor activity in the morning peak hours, the compressor is turned on some minutes before the morning standard electricity pricing period at 06:00 and stays on a few minutes into the standard electricity pricing period. This provides the cascade storage with sufficient gas to meet the demand without compressor activity past the morning peak electricity pricing time. Two compressor-on instances occur in the standard electricity pricing period between 10:00 and 18:00 which replenish the cascade storage sustaining the gas demand until after the night standard pricing period that ends at 22:00. A single compressor-on instance occurs in the subsequent off-peak period supplying gas for end of day demand. The profile accomplished by this approach reduces electricity cost for the CNG station in the low demand electricity pricing season from a baseline of R212.40 to R122.40 which is a $42.3 \%$ reduction in the day's electricity cost. This significant reduction in cost means that the energy cost reduction strategy is applicable throughout the year with significant savings in both electricity demand pricing seasons. 


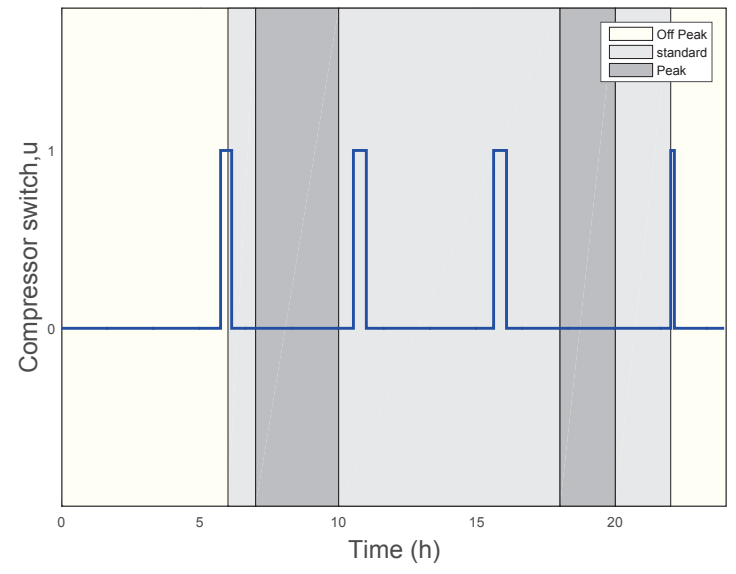

(a) Compressor switch action

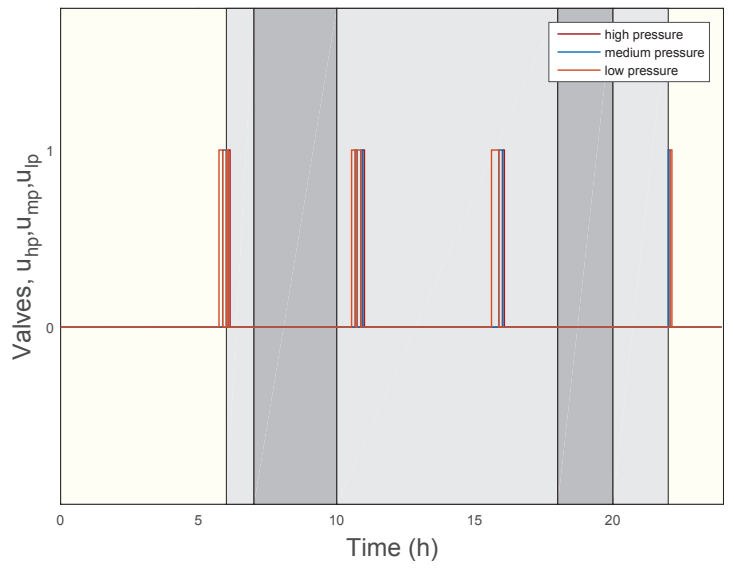

(b) Valve action

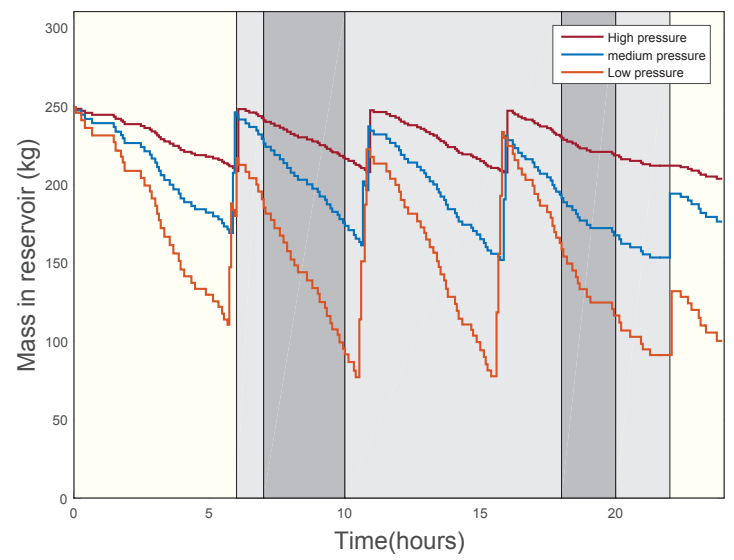

(c) Mass of gas in reservoir

Figure 6: System behaviour in the low demand electricity pricing season using the non-linear objective function method 


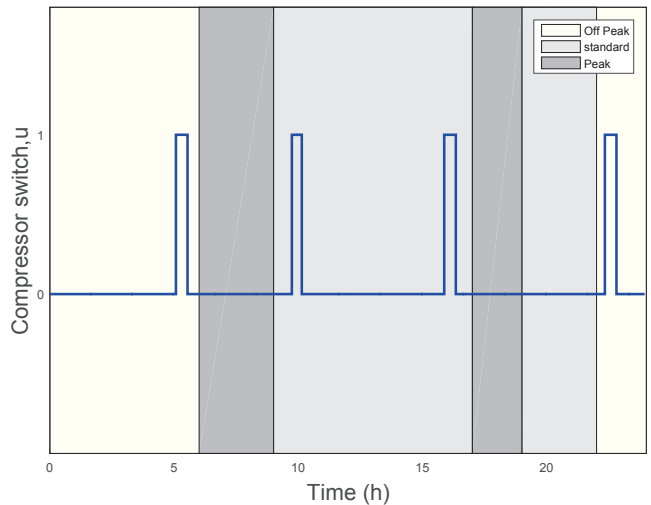

(a)

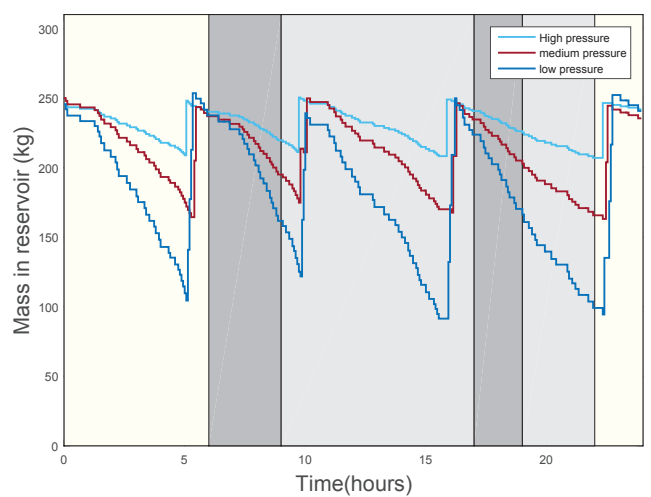

(b)

High electricity Demand season

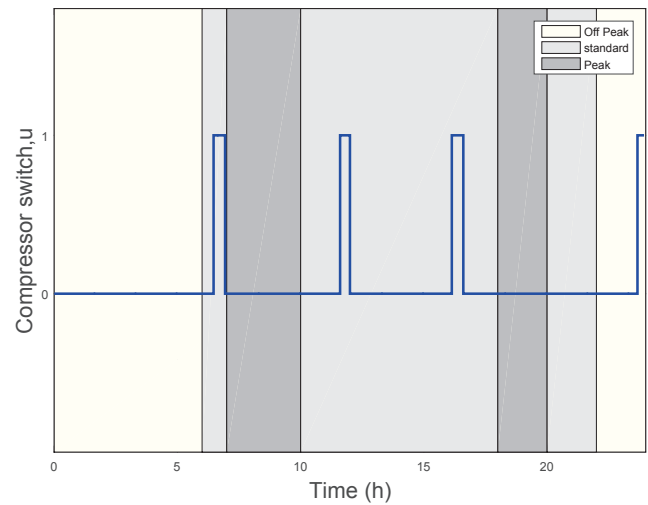

(a)

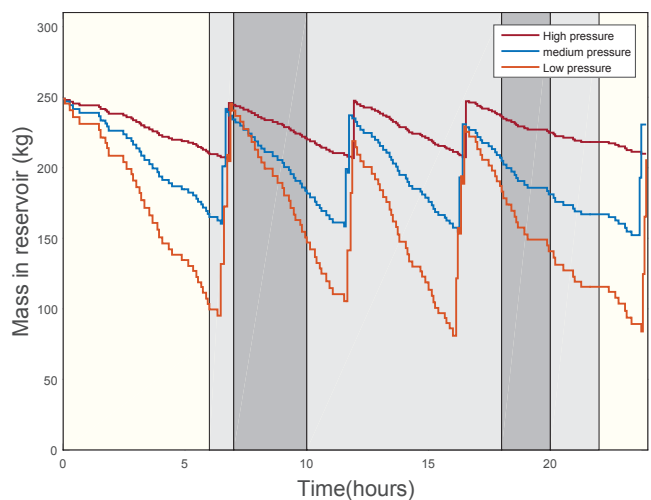

(b)

Low electricity demand season

Figure 7: (a) Compressor switching and (b) mass of gas in reservoir results with terminal constraints for high and low electricity demand seasons

\subsection{Solutions with terminal constraints}

It is evident from the Figures (3c), (4c), (5c) and (6c) that the mass of gas in the reservoir at the end of the 24 hour control horizon is different from the initial mass at the start of the control period under these strategies. This results in the initial conditions of the subsequent control period being different from those of the current one. When the terminal constraints are implemented with the non-linear objective function method of minimizing compressor switching frequency, the resulting profile of operation is shown in Figure (7). In this regime, the strategy is able to keep the compressor operations outside of the high electricity pricing periods as well as raise the quantity of gas in storage close to the initial condition levels while keeping the number of compressor instances at four. However the cost of electricity incurred rises to R171.60 for the high electricity demand season and R139.84 for the low electricity demand season due to the operation for restoring the levels of gas to initial conditions compared to optimization without the terminal constraints. The effect of the terminal constraints can be observed when the optimization is repeated for seven consecutive days shown in Figure (8). In general, the level of gas in storage 


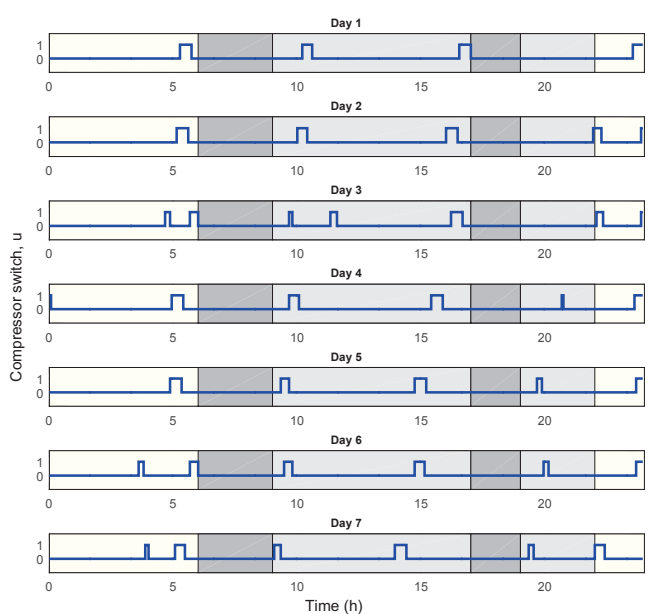

(a)

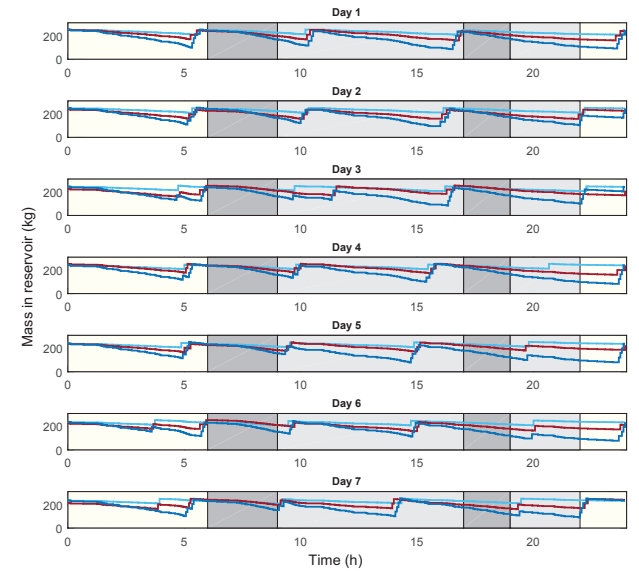

(b)

High electricity Demand season

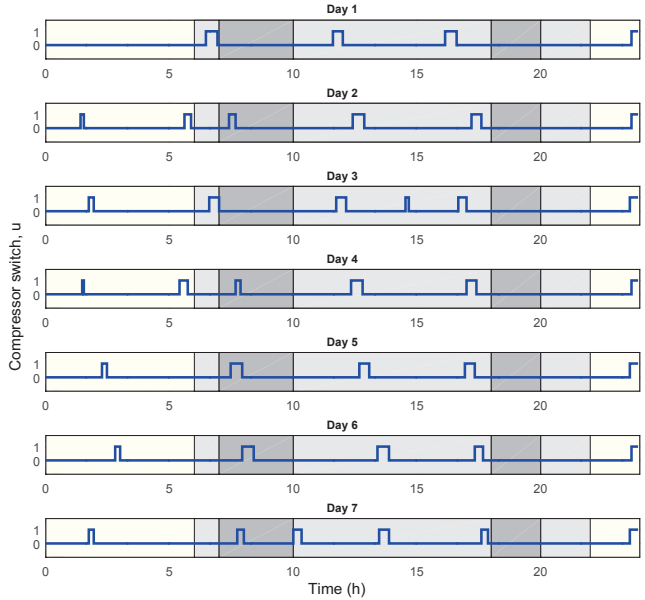

(a)

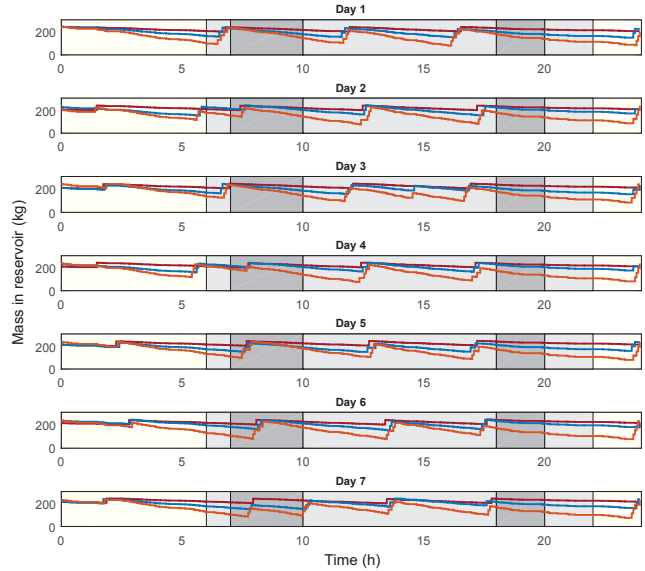

(b)

Figure 8: (a) Compressor switching and (b) mass of gas in reservoir results with terminal constraints for one week in high and low electricity demand seasons 
after the end of each day remains similar to the initial conditions for that day. However the small variations have an effect on the performance of the strategy with a tendency to increase the number of compressor switching instances in some of the days. A maximum of seven switching instances for a single day occur during the high demand electricity pricing season while a maximum of six occur during the low electricity pricing season. These number of switching instances are still 50\% lower than those observed under optimization without minimization of the switching frequency.

Over the course of the week in evaluation, the strategy is able to keep operation of the compressor outside the peak electricity pricing times for the high demand electricity pricing season. The resultant average cost of electricity is R176.13 per day, which represents $59.3 \%$ savings from the baseline. The highest cost at R179.91, happened on the fourth and seventh days while the lowest cost at R171.60 occurred on the first day. In the low demand electricity pricing season, the compressor is turned on during the morning peak electricity pricing time in the 4th, 5th, 6th and 7 th days. However, the strategy still manages to keep the average cost of electricity per day at R158.54 with the highest cost observed on the fifth day at R172.54 and the lowest on the first day at R143.16. The average savings on electricity cost for the low demand electricity pricing period is $25 \%$. The proposed strategy shows versatility in dealing with variations in initial conditions for consecutive days for the week under evaluation.

\section{Conclusion}

The use of compressed natural gas for the propulsion of motor vehicles can benefit greatly from optimization of the fuelling station operation by minimizing the energy cost. The present introductory work shows that $59.3 \%$ in electricity cost savings are achieved, while balancing the savings with a consideration for the life and reliability of the compressor. The subsequent savings translate to a reduction in compressor running cost by a 0.04 Rand cents margin per kilogram of gas sold in the low demand electricity pricing period and 0.23 Rand cents in the high demand electricity pricing period. These are significant margins that can allow compressed natural gas fuelling station operators to adjust the price of gas per unit of sale in order to attract more customers.

The control approach developed in this study can be used in setting operating schedules for compressors and priority panels in compressed natural gas stations operating in a time-based electricity tariff environment to save cost while prolonging the lifespan of the compressor by minimizing its frequent on/off switching. This can be done by changing the algorithm on the existing programmable logic controller that operates the compressor so that it is time-scheduled according to the results of the optimization, instead of the pressure limit cycled operation employed by the existing system. The main conditions arising from assumptions in this study that could affect the performance of the strategy in a real life scenario is a difference between the forecast and actual minimum and maximum temperatures over a control horizon which can result in unexpected changes in pressure. Safety interrupts for the maximum and minimum pressure can be implemented in the algorithm to deal with such cases where unpredictable events occur and expected optimized operation is violated. The safety interrupts can also deal with a variation in expected gas demand profile if it results in pressure limits in storage being reached.

The optimization model leads to improved economic efficiency of the compressed natural gas fuelling station and can reduce the contribution of the transportation industry to the emission 
of green house gases. This is because reduced costs have the potential to encourage consumer uptake of lower emission compressed natural gas powered vehicles over diesel and petrol vehicles. Further, the shifting of electric loads has the potential to reduce overall green house gas emission from the electricity generation infrastructure of the power utility. The outcome of the study justifies further optimization of the individual components of the fast-fill process to advance the goal of energy efficiency.

\section{Acknowledgement}

The authors thank the National Hub for Energy Efficiency and Demand Side Management at the University of Pretoria, South Africa for financial and other support.

\section{References}

[1] P. Bielaczyc, J. Woodburn, A. Szczotka, An assessment of regulated emissions and $\mathrm{CO}_{2}$ emissions from a European light-duty CNG-fueled vehicle in the context of Euro 6 emissions regulations, Applied Energy 117 (2014) 134-141.

[2] M. M. Movahed, H. B. Tabrizi, M. Mirsalim, Experimental investigation of the concomitant injection of gasoline and CNG in a turbocharged spark ignition engine, Energy Conversion and Management 80 (2014) 126-136.

[3] S. Yeh, An empirical analysis on the adoption of alternative fuel vehicles: the case of natural gas vehicles, Energy Policy 35 (11) (2007) 5865-5875.

[4] Y. Han, J. Kang, G. Zhang, Z. Liu, J. Tian, J. Chai, Performance evaluation of free piston compressor coupling organic Rankine cycle under different operating conditions, Energy Conversion and Management 86 (2014) 340-348.

[5] S. O. Akansu, Z. Dulger, N. Kahraman, T. N. Veziroglu, Internal combustion engines fueled by natural gashydrogen mixtures, International Journal of Hydrogen Energy 29 (14) (2004) 1527-1539.

[6] R. Amirante, E. Distaso, S. Di Iorio, P. Sementa, P. Tamburrano, B. Vaglieco, R. Reitz, Effects of natural gas composition on performance and regulated, greenhouse gas and particulate emissions in spark-ignition engines, Energy Conversion and Management 143 (2017) 338-347.

[7] E. A. Gilmore, A. Patwardhan, Passenger vehicles that minimize the costs of ownership and environmental damages in the Indian market, Applied energy 184 (2016) 863-872.

[8] G. Carle, Erdgasfahrzeuge und ihr beitrag zu einer $\mathrm{CO}_{2}$-reduktion im motorisierten personenverkehr der schweiz, Ph.D. thesis (2006).

[9] J. Ally, T. Pryor, Life-cycle assessment of diesel, natural gas and hydrogen fuel cell bus transportation systems, Journal of Power Sources 170 (2) (2007) 401-411.

[10] J. L. Kirk, A. L. Bristow, A. M. Zanni, Exploring the market for compressed natural gas light commercial vehicles in the United Kingdom, Transportation Research Part D: Transport and Environment 29 (2014) $22-31$.

[11] K. Ravindra, E. Wauters, S. K. Tyagi, S. Mor, R. Van Grieken, Assessment of air quality after the implementation of compressed natural gas (CNG) as fuel in public transport in Delhi, India, Environmental monitoring and assessment 115 (1-3) (2006) 405-417.

[12] W. Lin, N. Zhang, A. Gu, LNG (liquefied natural gas): A necessary part in China's future energy infrastructure, Energy 35 (11) (2010) 4383-4391.

[13] D. of Minerals, E. R. of South Africa, Gas infra-structure plan, Tech. rep., Department of Minerals and Energy Republic of South Africa (2005).

[14] I. Pretorius, S. Piketh, R. Burger, The impact of the South African energy crisis on emissions, Air Pollution XXIII; Longhurst, JWS, Brebbia, C., Brebbia, CA, Barnes, J., Eds (2015) 255-264.

[15] H. M. He, W. S. Lin, A. Z. Gu, Preliminary technical study on L/CNG stations, Natural Gas Industry 27 (4) (2007) 126.

[16] R. C. Elgin, C. L. Hagen, Development and operation of a self-refueling compressed natural gas vehicle, Applied Energy 155 (2015) 242-252. 
[17] M. Farzaneh-Gord, M. Deymi-Dashtebayaz, H. R. Rahbari, Studying effects of storage types on performance of CNG filling stations, Journal of Natural Gas Science and Engineering 3 (1) (2011) 334-340.

[18] S. Ramoutar, C. Riverol, A thermodynamic analysis of refueling a natural gas vehicle cylinder from a cascade reservoir using chilled natural gas, Journal of Natural Gas Science and Engineering 38 (2017) 298-322.

[19] K. Kountz, C. Blazek, W. Liss, A new natural gas dispenser control system, in: International Gas Research Conference, Vol. 4, Government Institutes Inc, 1998, pp. 135-145.

[20] B. F. Price, Apparatus and process for fast filling with natural gas, US Patent 5,370,159 (Dec. 6 1994).

[21] R. E. Knowlton, Natural gas storage and retrieval system, US Patent 4,805,674 (Feb. 21 1989).

[22] A. M. Barajas, J. C. Buckingham, S. Svedeman, System for controlling the fill of compressed natural gas cylinders, US Patent 5,868,176 (Feb. 9 1999).

[23] Y. Han, X. D. You, X. Zhang, H. Y. Wang, C. Deng, A novel design of the CNG dispenser electronic control system, in: Applied Mechanics and Materials, Vol. 448, Trans Tech Publ, 2014, pp. 3119-3122.

[24] C.-1. Su, J.-1. Liu, D.-1. Qiu, J.-y. Cai, S.-s. Li, Intelligent compressed natural gas dispensers, China Measurement \& Testing Technology 6 (2008) 041.

[25] C. D. Emmer, J. Gamble, C. Zelasko, T. K. Drube, Liquid and compressed natural gas dispensing system, US Patent 7,069,730 (Jul. 4 2006).

[26] A. Gram, M. Ursan, Combined liquefied gas and compressed gas re-fueling station and method of operating same, US Patent 7,284,575 (Oct. 23 2007).

[27] M. Tsuchiya, H. Ogasa, H. Matsuura, H. Shimanuki, I. Fujii, Thermodynamic behavior of supply gas and influence on vehicle fuel fill line during CNG fast fill, Tech. rep., SAE Technical Paper (1996).

[28] G. Thomas, J. Goulding, C. Munteam, Measurement, approval and verification of CNG dispensers, NWML KT11 Report.

[29] M. Saadat-Targhi, J. Khadem, M. Farzaneh-Gord, Thermodynamic analysis of a CNG refueling station considering the reciprocating compressor, Journal of Natural Gas Science and Engineering 29 (2016) 453-461.

[30] S. Mokhatab, W. A. Poe, Handbook of natural gas transmission and processing, Gulf professional publishing, 2012.

[31] J. Xueqin, Study of urban CNG station location arrangement and the size, Public Utilities 1 (2007) 005.

[32] X. Xia, L. Zhang, Industrial energy systems in view of energy efficiency and operation control, Annual Reviews in Control 42 (2016) 299-308.

[33] X. Xia, J. Zhang, W. Cass, Energy management of commercial buildings-a case study from a POET perspective of energy efficiency, Journal of Energy in Southern Africa 23 (1) (2012) 23-31.

[34] X. Xia, J. Zhang, Modeling and control of heavy-haul trains [applications of control], IEEE Control Systems 31 (4) (2011) 18-31.

[35] X. Xia, J. Zhang, Energy efficiency and control systems-from a POET perspective, IFAC Proceedings Volumes 43 (1) (2010) 255-260.

[36] X. Zhuan, X. Xia, Optimal operation scheduling of a pumping station with multiple pumps, Applied Energy 104 (2013) 250-257.

[37] X. Xia, J. Zhang, Mathematical description for the measurement and verification of energy efficiency improvement, Applied Energy 111 (2013) 247-256.

[38] X. Zhuan, X. Xia, Development of efficient model predictive control strategy for cost-optimal operation of a water pumping station, IEEE Transactions on Control Systems Technology 21 (4) (2013) 1449-1454.

[39] X. Ye, X. Xia, J. Zhang, Optimal sampling plan for clean development mechanism energy efficiency lighting projects, Applied energy 112 (2013) 1006-1015.

[40] K. J. Kountz, Modeling the fast fill process in natural gas vehicle storage cylinders, Tech. rep., Institute of Gas Technology, Chicago, IL (United States) (1994).

[41] M. Farzaneh-Gord, Compressed natural gas-single reservoir filling process, International Gas Enginering and Management 48 (6) (2008) 16-18.

[42] M. Farzaneh-gord, S. Hashemi, A. Farzaneh-kord, Thermodynamics analysis of cascade reserviors filling process of natural gas vehicle cylinders 1.

[43] F. Olmos, V. I. Manousiouthakis, Gas tank fill-up in globally minimum time: Theory and application to hydrogen, International Journal of Hydrogen Energy 39 (23) (2014) 12138-12157. 
[44] M. Deymi-Dashtebayaz, M. F. Gord, H. R. Rahbari, Studying transmission of fuel storage bank to NGV cylinder in CNG fast filling station, Journal of the Brazilian Society of Mechanical Sciences and Engineering 34 (4) (2012) 429-435.

[45] J. Khadem, M. Saadat-Targhi, M. Farzaneh-Gord, Mathematical modeling of fast filling process at CNG refueling stations considering connecting pipes, Journal of Natural Gas Science and Engineering 26 (2015) 176-184.

[46] K. J. Kountz, C. F. Blazek, F. Christopher, NGV fuelling station and dispenser control systems, Tech. rep., report GRI-97/0398, Gas Research Institute, Chicago, Illinois, November (1997).

[47] P. Stouffs, M. Tazerout, P. Wauters, Thermodynamic analysis of reciprocating compressors, International Journal of Thermal Sciences 40 (1) (2001) 52-66.

[48] S. Y. Sun, T.-R. Ren, New method of thermodynamic computation for a reciprocating compressor: computer simulation of working process, International Journal of Mechanical Sciences 37 (4) (1995) 343-353.

[49] M. Elhaj, F. Gu, A. Ball, A. Albarbar, M. Al-Qattan, A. Naid, Numerical simulation and experimental study of a two-stage reciprocating compressor for condition monitoring, Mechanical Systems and Signal Processing 22 (2) (2008) 374-389.

[50] D. Ndiaye, M. Bernier, Dynamic model of a Hermetic reciprocating compressor in on-off cycling operation (abbreviation: Compressor dynamic model), Applied Thermal Engineering 30 (8) (2010) 792-799.

[51] M. Frick, K. W. Axhausen, G. Carle, A. Wokaun, Optimization of the distribution of compressed natural gas (CNG) refueling stations: Swiss case studies, Transportation Research Part D: Transport and Environment 12 (1) (2007) 10-22.

[52] M. Malakoutirad, T. H. Bradley, C. Hagen, Design considerations for an engine-integral reciprocating natural gas compressor, Applied Energy 156 (2015) 129-137.

[53] H. J. Bang, S. Stockar, M. Muratori, G. Rizzoni, Modeling and analysis of a CNG residential refueling system, in: ASME 2014 Dynamic Systems and Control Conference, no. DSCC2014-6160, American Society of Mechanical Engineers, 2014.

[54] J. Jannati, D. Nazarpour, Optimal energy management of the smart parking lot under demand response program in the presence of the electrolyser and fuel cell as hydrogen storage system, Energy Conversion and Management 138 (2017) 659-669.

[55] M. H. Albadi, E. El-Saadany, A summary of demand response in electricity markets, Electric Power Systems Research 78 (11) (2008) 1989-1996.

[56] L. Zhang, X. Xia, J. Zhang, Improving energy efficiency of cyclone circuits in coal beneficiation plants by pump-storage systems, Applied Energy 119 (2014) 306-313.

[57] K. Herter, P. McAuliffe, A. Rosenfeld, An exploratory analysis of California residential customer response to critical peak pricing of electricity, Energy 32 (1) (2007) 25-34.

[58] D. Setlhaolo, X. Xia, J. Zhang, Optimal scheduling of household appliances for demand response, Electric Power Systems Research 116 (2014) 24-28.

[59] U. E. Ekpenyong, J. Zhang, X. Xia, Mathematical modelling for the social impact to energy efficiency savings, Energy and Buildings 84 (2014) 344-351.

[60] Z. Wu, H. Tazvinga, X. Xia, Demand side management of photovoltaic-battery hybrid system, Applied Energy 148 (2015) 294-304.

[61] N. L. Newhouse, W. E. Liss, Fast filling of NGV fuel containers, Tech. rep., SAE Technical Paper (1999).

[62] H. H. Nguyen, V. Uraikul, C. W. Chan, P. Tontiwachwuthikul, A comparison of automation techniques for optimization of compressor scheduling, Advances in Engineering Software 39 (3) (2008) 178-188.

[63] E. M. Wanjiru, X. Xia, Energy-water optimization model incorporating rooftop water harvesting for lawn irrigation, Applied Energy 160 (2015) 521-531.

[64] E. Heidaryan, J. Moghadasi, M. Rahimi, New correlations to predict natural gas viscosity and compressibility factor, Journal of Petroleum Science and Engineering 73 (1) (2010) 67-72.

[65] A. Bahadori, S. Mokhatab, B. F. Towler, Rapidly estimating natural gas compressibility factor, Journal of Natural Gas Chemistry 16 (4) (2007) 349-353.

[66] P. F. Hopkins, Method and apparatus for dispensing compressed gas into a vehicle, US Patent 5,406,988 (Apr. 18 1995).

[67] H. H. Nguyen, C. W. Chan, Applications of artificial intelligence for optimization of compressor scheduling, 
Engineering Applications of Artificial Intelligence 19 (2) (2006) 113-126.

[68] H. P. Bloch, J. J. Hoefner, Reciprocating Compressors:: Operation and Maintenance, Gulf Professional Publishing, 1996.

[69] E. Giacomelli, F. Bernardini, A. Pini, H. Andree, Application of gas turbines for large reciprocating compressor drive, in: ASME 1989 International Gas Turbine and Aeroengine Congress and Exposition, American Society of Mechanical Engineers, 1989, pp. V004T10A006-V004T10A006.

[70] P. Walsh, J. Lamancusa, A variable stiffness vibration absorber for minimization of transient vibrations, Journal of sound and vibration 158 (2) (1992) 195-211.

[71] W. Booysen, M. Kleingeld, J. Van Rensburg, Optimising compressor control strategies for maximum energy savings, ICUE, Cape Town (2009) 10-12.

[72] C. K. Sun, V. Uraikul, C. W. Chan, P. Tontiwachwuthikul, An integrated expert system/operations research approach for the optimization of natural gas pipeline operations, Engineering Applications of Artificial Intelligence 13 (4) (2000) 465-475.

[73] T. Mathaba, X. Xia, J. Zhang, Analysing the economic benefit of electricity price forecast in industrial load scheduling, Electric Power Systems Research 116 (2014) 158-165.

[74] E. M. Wanjiru, L. Zhang, X. Xia, Model predictive control strategy of energy-water management in urban households, Applied Energy 179 (2016) 821-831.

[75] A. M. Bagirov, A. Barton, H. Mala-Jetmarova, A. Al Nuaimat, S. Ahmed, N. Sultanova, J. Yearwood, An algorithm for minimization of pumping costs in water distribution systems using a novel approach to pump scheduling, Mathematical and Computer Modelling 57 (3) (2013) 873-886.

[76] B. P. Numbi, J. Zhang, X. Xia, Optimal energy management for a jaw crushing process in deep mines, Energy 68 (2014) 337-348.

[77] T. Faulwasser, M. Korda, C. N. Jones, D. Bonvin, On turnpike and dissipativity properties of continuous-time optimal control problems, Automatica 81 (2017) 297-304.

[78] H. Zhang, F. Zhao, K. Fang, J. W. Sutherland, Energy-conscious flow shop scheduling under time-of-use electricity tariffs, CIRP Annals-Manufacturing Technology 63 (1) (2014) 37-40.

[79] S. M. Baladi, J. A. Herriges, T. J. Sweeney, Residential response to voluntary time-of-use electricity rates, Resource and Energy Economics 20 (3) (1998) 225-244.

[80] K. J. Kountz, W. E. Liss, C. F. Blazek, Automated process and system for dispensing compressed natural gas, US Patent 5,810,058 (Sep. 22 1998).

[81] A. M. Bagirov, A. Barton, H. Mala-Jetmarova, A. Al Nuaimat, S. Ahmed, N. Sultanova, J. Yearwood, An algorithm for minimization of pumping costs in water distribution systems using a novel approach to pump scheduling, Mathematical and Computer Modelling 57 (3) (2013) 873-886.

[82] R. Allan, R. Billinton, M. De Oliveira, Reliability evaluation of electrical systems with switching actions, in: Proceedings of the Institution of Electrical Engineers, Vol. 123, IET, 1976, pp. 325-330.

[83] M. Bosman, Availability analysis of a natural gas compressor plant, Reliability engineering 11 (1) (1985) 13-26.

[84] H. X. Wang, M. He, L. Yang, N. Li, Y.-d. Wang, Suggestions on relevant design codes for CNG filling stations in China, Gas \& Heat 12 (2009) 027.

[85] H. He, H. Daume III, J. M. Eisner, Learning to search in branch and bound algorithms, in: Advances in Neural Information Processing Systems, 2014, pp. 3293-3301. 